\title{
Doppler Modulation of X-ray lines in Cygnus X-3
}

\author{
Michael J. Stark and Malinda Saia \\ Department of Physics, Lafayette College, Easton, PA 18042 \\ starkm@lafayette.edu, saiam@lafayette.edu
}

\begin{abstract}
We measured Doppler shifts of three bright spectral lines in the X-ray emission from Cygnus $\mathrm{X}-3$ as recorded by the Chandra X-Ray Observatory. Doppler shifts of lines associated with Si XIV and S XVI exhibit orbital modulation. The magnitude and phasing of this modulation relative to the orbital ephemeris indicate the location of the source of this emission within the wind emanating from the compact object's companion. These observations enable us to make an indirect measurement of the separation of the two stars. Under certain assumptions our observation of a line associated with Fe XXV also limits the mass of the compact object $M_{\mathrm{C}} \leq$ $3.6 M_{\odot}$.

Subject headings: X-rays: binaries — binaries:close — stars: individual (Cygnus X-3)
\end{abstract}

\section{Introduction}

In spite of near continuous study at many wavelengths since its discovery (Giacconi et al. 1967), the nature of the Cygnus X-3 binary system remains mysterious. Several important questions remain about the nature of the compact object and its companion and about the specific location of the source of observed X-ray and infrared emission. Existing models describe the system either as a high-mass system consisting of a black hole and a Wolf-Rayet star (Cherepashchuk \& Moffat 1994) or as a low-mass system consisting of a neutron star and a degenerate companion (Tavani, Ruderman, \& Shaham 1989). Both of these models account for the intense X-ray emission, the existence of a Helium-rich stellar wind, and the resulting large mass loss from the system.

The observation of a $12.6 \mathrm{~ms}$ pulsar signal in gamma ray data (Chadwick et al. 1985) is evidence that the compact object is a neutron star. However, though this pulsar signal has been repeatedly observed by the group that first reported it (Bowden et al. 1992), it has not been confirmed by independent investigators and it has never been observed at lower energies. Non-observation at lower energies is not incompatible with observation at higher energies since the scattering dis- tance for X-rays in the dense wind is much shorter than for gamma rays. If true, the pulsar detection is proof that the compact object is a neutron star. Absent independent confirmation, however, the reports of a pulsar signal must be regarded with skepticism.

One key to understanding the nature of the two stars in the system would be a direct measurement of their masses. By measuring the Doppler shift of the infrared emission, Schmutz et al. (1996) report that they have measured the velocity of the infrared companion and constrained the compact object to be, at the least, a very massive neutron star. Hanson, Still, \& Fender (2000) use the Doppler shift of a different infrared feature to make a more correct measurement of the velocity of the companion which prefers lower mass systems except that it also implies that the companion must be the dominant mass in the system. This rules out systems consisting of a neutron star with a white dwarf companion. Knowing the velocity of only one of the stars of a binary system does not constrain the total mass of the system. To determine the masses of both stars, we must make a measurement of the velocity of the compact object. 


\section{Observations}

We used data collected by the Chandra X-Ray Observatory $(C X O)$ during the radio quiet period prior to the April 2000 radio outburst (McCollough et al. 2000). Data were taken on two occasions two days apart and each observation lasted approximately five hours, completely covering one orbital period of the source. The observations were carried out using the High Energy Transmission Grating Spectrometer (HETGS). Due to photon pileup in the Advanced CCD Imaging Spectrometer caused by the brightness of Cygnus X3 , the automatic processing was unable to pinpoint the center of the zeroth order maximum of the grating spectra. We refined the automatically determined source location by fitting prominent spectral features in both the positive and negative first order spectra of the Medium Energy Grating (MEG) and the High Energy Grating (HEG). The source location was adjusted so that the fitted wavelengths for all four first order spectra were identical. By this method, the location of the Xray source was determined to be $20^{\mathrm{h}} 32^{\mathrm{m}} 25.8 \pm 0.1$ $+40^{\circ} 57^{\prime} 28^{\prime \prime} .0 \pm 0^{\prime \prime} .1$ which, given the $0^{\prime \prime} 6$ absolute astrometric accuracy of the $C X O$, is consistent with the radio location given by Ogley, Bell Burnell, \& Fender (2001).

We divided the data into eight bins by the orbital phase of the source according to the ephemeris in Table 1 . In each of these phase bins, we fit a continuum model and Gaussian line profiles to data from both positive and negative first-order spectra from the HEG and the MEG simultaneously. The continuum was fit by a power law spectrum with a model of interstellar absorption. Though the distance to Cygnus X-3 is still poorly determined, the degree of the absorption fit by the spectrum, $8.5 \pm 0.1 \times 10^{22} \mathrm{H} \mathrm{I} / \mathrm{cm}^{2}$ is consistent with other measurements of the distance to Cygnus X-3 or of the intervening absorption column (Serlemitsos et al. 1975; Dickey 1983; Predehl et al. 2000). Our measured value is also independent of orbital phase.

We fit a Gaussian line function to the three most prominent lines visible in the spectrum, the Lyman $\alpha$ lines of Si XIV and S XVI and the Helium $\alpha$ line of Fe XXV. Only these three lines were strong enough in the phase-dependent spectra that they could be fit reliably for all phase intervals.
Examples of these fits are shown in Figure 1. The Si XIV and S XVI lines showed a consistent average Doppler shift corresponding to a recession velocity of $\sim 550 \mathrm{~km} / \mathrm{s}$. This is somewhat less than the velocity of $750-800 \mathrm{~km} / \mathrm{s}$ reported by Paerels et al. (2000), also from HETGS data, but nearly consistent given the absolute spectral accuracy of the HETGS is on the order of $100 \mathrm{~km} / \mathrm{s}$.

Figure 2(a) shows the Doppler shift of the $6.18 \AA$ Lyman $\alpha$ line of Si XIV and Figure 2(b) shows the Doppler shift of the $4.73 \AA$ Lyman $\alpha$ line of S XVI. The parameters of the best fit of a cosine function to the Doppler shift of each line are given in Table 2. The sinusoidal function provides a better fit to the data than does a constant function. For Si XIV, $\chi^{2}$ per degree of freedom drops from 2.7 to 1.6 with the change from a constant function to a sinusoidal function. In S XVI the change in $\chi^{2}$ per degree of freedom is from 2.8 to 1.5. The F-test probabilities for these detections are $18 \%$ and $14 \%$, respectively, so the chance probability of the simultaneous detection of these two modulations is $2.5 \%$.

The Doppler shift of the Si XIV line has significant day-to-day variation though the modulation is clearly present on both days. The modulation of the S XVI line appears to be particularly repeatable. Sinusoidal functions may not be the most appropriate functional forms to fit to these data. In the simplest configuration, the emission region will be in the form of a ring or a disk near the compact object sampling different wind velocities. In addition, different parts of the emission region may be occulted at different times. Beyond the apparent inadequacy of the shape of the fit function, the phase of the maximum Doppler shift may be affected by the differential occultation of the emission region. Nevertheless, the amplitude of the fit sinusoid gives a reasonable approximation of the amplitude of the correct functional form. The quality of these fits to the data $\left(\chi^{2}\right.$ per degree of freedom is 1.6 for Si XIV and 1.5 for S XVI) indicate that within our uncertainties the true functional form is not much more complicated than a sinusoid.

Figure 2(c) shows the Doppler shift of the $1.86 \AA \mathrm{Fe}$ XXV He $\alpha$ line. The data are consistent with a constant Doppler shift. The $\chi^{2}$ per degree of freedom assuming a constant Doppler shift is 1.77 while the best fit sinusoidal function 
TABLE 1

Cygnus X-3 orbital ephemeris

\begin{tabular}{c}
\hline$T_{n}=T_{0}+P_{0} n+P_{0} \dot{P} n^{2} / 2$ \\
\hline$T_{0}=$ JD $2440949.89016 \pm 0.00064$ \\
$P_{0}=0.19968462 \pm 6 \times 10^{-8} \mathrm{~d}$ \\
$\dot{P}=(5.52 \pm 0.12) \times 10^{-10}$ \\
\hline
\end{tabular}

Note. $-T_{n}$ are the times of successive X-ray minima from Stark \& Saia, in preparation.

TABLE 2

Doppler Shift of Strong Emission Lines

\begin{tabular}{lcccccc}
\hline \hline Line & $\begin{array}{c}\text { Source } \\
\text { Wavelength } \\
(\AA)\end{array}$ & $\begin{array}{c}\text { Observed } \\
\text { Wavelength } \\
(\AA)\end{array}$ & $\begin{array}{c}\text { Recession } \\
\text { Velocity } \\
(\mathrm{km} / \mathrm{s})\end{array}$ & $\begin{array}{c}\text { Modulation } \\
\text { Amplitude } \\
(\AA)\end{array}$ & $\begin{array}{c}\text { Modulation } \\
\text { Amplitude } \\
(\mathrm{km} / \mathrm{s})\end{array}$ & $\begin{array}{c}\text { Phase } \\
\text { Offset }^{\mathrm{b}} \\
\left({ }^{\circ}\right)\end{array}$ \\
\hline Si XIV & 6.1822 & $6.19381 \pm 0.00058$ & $563 \pm 28$ & $0.0027 \pm 0.0007$ & $133 \pm 35$ & $21 \pm 14$ \\
S XVI & 4.7292 & $4.73774 \pm 0.00045$ & $541 \pm 29$ & $0.0025 \pm 0.0006$ & $158 \pm 35$ & $-37 \pm 14$ \\
Fe XXV & $1.8617^{\mathrm{c}}$ & $1.86340 \pm 0.00038$ & $274 \pm 61^{\mathrm{c}}$ & $<0.0014^{\mathrm{d}}$ & $<220^{\mathrm{d}}$ & $\cdots$ \\
\hline
\end{tabular}

${ }^{a}$ Quoted uncertainty is from the fit of a Gaussian line profile. Absolute spectral accuracy of the HETGS is on the order of $100 \mathrm{~km} \mathrm{~s}^{-1}$.

${ }^{b}$ Phase offset is for the maximum red-shift relative to the time of the minimum intensity of the X-ray continuum.

${ }^{\mathrm{c}}$ The reported Fe XXV source wavelength is from a combination of lines we may have modeled incorrectly. The value of the recession velocity is, therefore, subject to a large, unknown systematic error. It is not, however, important to the results presented here.

${ }^{\mathrm{d}}$ Upper limits are $90 \%$ confidence limits. All other uncertainties are $1 \sigma$ 


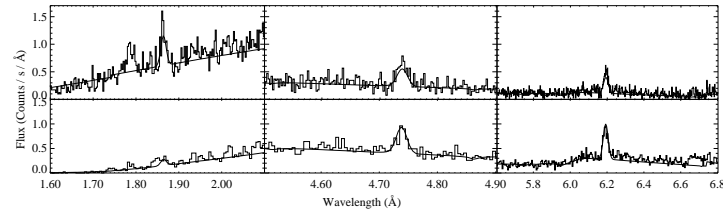

Fig. 1.- Examples of line fitting from this analysis. The fits indicated here by the thick line are for the phase interval centered on 0.625 on 2000 April 4, The left panels are for FE XXV, the center panels are for S XVI and the right panels are for Si XIV. The top panels are from the positive first order HEG spectrum and the bottom panels are from the positive first-order MEG spectrum. The fitted profiles are derived from fitting all four of the first-order spectra simultaneously.

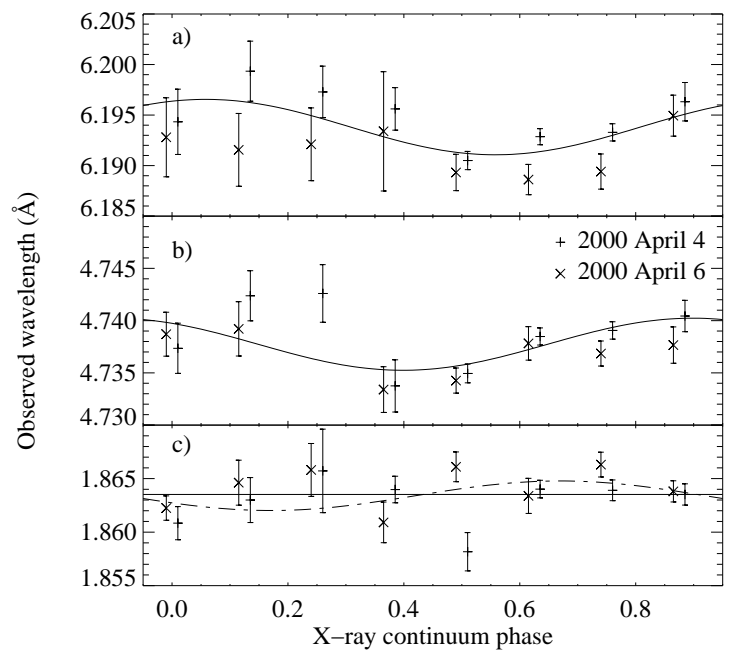

Fig. 2.- Observed wavelength of (a) the Si XIV Ly $\alpha$ line and (b) the S XVI Ly $\alpha$ line and (c) the Fe XXV He $\alpha$ line as a function of the X-ray phase. The S XVI point at phase 0.25 from 2000 April 6 is corrupted by a peculiarity of the background and is excluded from the fit. The phases on the plots have been shifted slightly to reveal overlapping data points. The dashed line in (c) corresponds to the $90 \%$ confidence upper limit on the modulation of the Fe XXV line. yields a $\chi^{2}$ per degree of freedom of 1.84. The non-observation of a modulation of the Doppler shift in Fe XXV allows us to set an upper limit on the velocity of the emission region for this line. The $90 \%$ confidence upper limit derived for a fit to these data is $220 \mathrm{~km} / \mathrm{s}$.

\section{Discussion}

In order to use our observations to draw conclusions about the size and mass of the Cygnus X-3 system, we must first decide where in the system the emission is produced. van Kerkwijk (1993) observed that line emission from hydrogen-like helium was produced in that part of the stellar wind of the companion which is shaded by the companion from the X-rays emanating from the compact object. According to his model, the rest of the wind is too highly ionized to produce helium line emission. The coincidence between the maximum red shift of the line emission from hydrogen-like silicon and sulfur and the time of the X-ray minimum (Taken to indicate the superior conjunction of the compact object) suggests that these emission features are produced in the companion wind near the compact object. The fact that the silicon and sulfur emission are produced much nearer to the X-ray source than the hydrogen emission suggests that the dominant source of ionization in the Cygnus X-3 system is the X-rays. The level of ionization appears to increase with proximity to the source of X-rays. Emission from hydrogenlike and helium-like iron will thus be produced even closer to the X-ray source-presumably the compact object - than is the silicon or sulfur emission. We will, therefore, assume that the emission feature produced by helium-like iron is produced close to the compact object.

\subsection{Stellar Mass}

The emission features of highly ionized iron may be produced in a region of the wind captured by the compact object or from an accretion disk around the object if not from the surface of the object itself. Without knowing the phase relationship between the Doppler shift of the Fe XXV line and the X-ray ephemeris, we can not be sure that it would reveal the motion of the compact object. If, however, we assume that this is so we can use our non-observation of Doppler shift in the 
Fe XXV line to constrain the velocity of the compact object. The equation for the mass function

$$
m_{f}=\frac{1}{2 \pi G} P v_{\mathrm{Max}}^{3}\left(1-\epsilon^{2}\right)^{3 / 2}
$$

yields an upper limit on the mass function of $0.22 M_{\odot}$ taking $P=4.8 \mathrm{~h}$ and our $90 \%$ confidence upper limit of $220 \mathrm{~km} / \mathrm{s}$ for the maximum radial velocity, $v_{\text {Max }}$. Ghosh et al. (1981) show that a high mass-loss rate can drive the system into an eccentric orbit as well as cause it to circularize. For simplicity we will assume $\epsilon=0$ for the time being.

By measuring the Doppler shift of the centroid of an infrared absorption feature in the stellar wind Hanson et al. (2000) calculated a mass function for the wind producing companion of $0.027 M_{\odot}$. We can combine this mass function with our mass function upper limit by simultaneously solving the following equations

$$
m_{f_{\mathrm{D}}}=\frac{\left(M_{\mathrm{C}} \sin i\right)^{3}}{\left(M_{\mathrm{D}}+M_{\mathrm{C}}\right)^{2}}, m_{f_{\mathrm{C}}}=\frac{\left(M_{\mathrm{D}} \sin i\right)^{3}}{\left(M_{\mathrm{C}}+M_{\mathrm{D}}\right)^{2}}
$$

Where the subscript $\mathrm{C}$ refers to the compact object and the subscript $\mathrm{D}$ refers to the mass donating companion. $i$ is the inclination of the binary orbit. The upper limits on the masses of the two stars derived this way are $M_{\mathrm{C}} \leq 0.24 M_{\odot}$ and $M_{\mathrm{D}} \leq 0.49 M_{\odot}$ for an inclination, $i=90^{\circ}$. These limits essentially exclude all stars that could produce the observed X-ray flux. Mass upper limits as a function of orbital inclinations are shown in Figure 3(a).

The orbital model of Cygnus X-3 that has been used most extensively is one of two stellar wind models proposed by Ghosh et al. (1981) which have a large orbital inclination and a large eccentricity. Even Hanson et al. (2000) state that the inclination should be large, but our results, taken in combination with theirs, make large inclinations unphysical. In light of other recent work (Singh et al. 2002) it appears that the lack of apsidal motion in the system requires that the orbital eccentricity be small. In addition, several authors (van der Klis \& Bonnet-Bidaud 1989; Mioduszewski et al 2001) demonstrate that the orbital inclination of the system must also be small. The best fit to the Ghosh et al. (1981) model based on a variable luminosity X-ray source inside its companion's stellar wind satisfies the need for both a small orbital inclination $\left(i=24^{\circ}\right)$ and eccentricity $(\epsilon=0.14)$. Using these values in Equations $1 \& 2$ gives an upper limit for the mass of the compact object, $M_{\mathrm{C}} \leq 3.6 M_{\odot}$ and for the companion of $M_{\mathrm{D}} \leq 7.3 M_{\odot}$. These mass limits don't preclude the possibility of the system being composed of a black hole and a Wolf-Rayet star. Neither do they rule out the compact object being a neutron star but the suggestion that its companion is more massive than the compact object rules out the possibility that the companion is also degenerate (a white dwarf).

\subsection{Stellar Size}

Table 2 shows that the maximum red shift of the Si XIV and S XVI emission lines are nearly coincident with the minimum of the continuum $\mathrm{X}$-ray intensity. The coincidence of the maximum radial velocity of the line emission and the X-ray minimum means that the line emitting region is moving most directly away from Earth at the same time that the compact object is at its furthest distance from Earth. This, then, suggests that the line emission emanates from a region of the companion's stellar wind blowing by the compact object and close to it. We can therefore use our measured velocity for this wind with a model of wind acceleration to constrain the size of the binary system.

The standard model of stellar wind acceleration appropriate to Wolf-Rayet stars is

$$
v(r)=v_{\infty}\left(1-\frac{r_{c}}{r}\right)^{\beta}
$$

where $r_{c}$ is the core radius of the star and $\beta$ expresses the degree of acceleration. Alternately, the wind velocity can be expressed in terms of the extent of the accelerating wind,

$$
v(r)=v_{\infty}\left(\frac{r}{r_{\infty}}\right)^{\gamma}
$$

(Cherepashchuk \& Moffat 1994) where $r_{\infty}$ is the distance from the wind producing star to where the terminal velocity is reached. We can combine the wind velocities implied by the analysis of the Si XIV and S XVI lines to provide an estimate of the wind velocity in the emission region surrounding the compact object of $146 \pm 50 \mathrm{~km} / \mathrm{s}$. Taking $v_{\infty}=1500 \mathrm{~km} / \mathrm{s}$ (Schmutz et al. 1996), 
$r_{\infty}=15 R_{\odot}$ (Fender, Hanson \& Pooley 1999), and $\gamma=1$ (Antokhin \& Cherepashchuk 2001), the distance between the emitting region and the wind producing companion star is $1.5 \pm 0.5 R_{\odot}$. This result assumes an inclination $i=90^{\circ}$. For an inclination angle $i=24^{\circ}$, we calculate $3.6 \pm 1.2 R_{\odot}$ for the distance between the donor star and the emission region. Since this emission comes from near the X-ray star, the distance calculated between the emission region and the companion star is the distance between the two stars.

The size of the Roche lobe of each star can be calculated directly from the mass functions. The radius of the Roche lobes is given by

$$
\frac{r_{\mathrm{L}}}{a}=\frac{0.49 q^{2 / 3}}{0.6 q^{2 / 3}+\ln \left(1+q^{1 / 3}\right)}
$$

(Eggleton 1983) where $a$ is the binary separation and $q$ is the ratio of the stellar masses, which is only dependent on the mass functions and which in the case of the results presented here $M_{\mathrm{D}} / M_{\mathrm{C}}=$ 2.0. This yields a Roche lobe radius of the companion star of $R_{\mathrm{D}}=1.6 \pm 0.5 R_{\odot}$ at an inclination, $i=24^{\circ}$, which is somewhat small for a WolfRayet star (Moffat \& Marchenko 1996) especially given that the dominant mass-transfer process is the stellar wind rather than Roche Lobe overflow. The companion Roche lobe radius is plotted as a function of the inclination of the system in Figure $3(\mathrm{~b})$.

\section{Conclusion}

We have determined an upper limit on the radial motion of the X-ray emitting star. This limit assumes that the Doppler shift of the emission feature produced by Fe XXV would reveal the motion of the X-ray emitting star. The relative phases of the observed Doppler shift of the He II, Si XIV, and S XVI emission features suggest that the degree of ionization of the circumstellar material is correlated with proximity to the X-ray source. This is consistent with our assumption. Combined with several other observations, we begin to paint a consistent picture of the Cygnus X-3 system. The picture favored by our analysis is of a system consisting of two relatively low mass stars, a compact object $M_{\mathrm{C}} \leq 3.6 M_{\odot}$ and its companion $M_{\mathrm{D}} \leq 7.3 M_{\odot}$ which orbit each other separated by $3.6 \pm 1.2 R_{\odot}$ in relatively circular $\epsilon=0.14$ orbit that has a small inclination $i=24^{\circ}$ relative to our line of site. Future X-ray observations will enable us to test the assumptions underlying this picture and, if they prove correct, to determine the orbital parameters of the system.

\section{REFERENCES}

Antokhin, I. I. \& Cherepashchuk, A. M. 2001, AZh, 78, 313

Bowden, C. C. G. et al. 1992, J. Phys. G: Nucl. Part. Phys., 18, 413

Chadwick, P. M. et al. 1985, Nature, 318, 642

Cherepashchuk, A. M., \& Moffat, A. F. J. 1994, ApJ, 424, L53

Dickey, J. M. 1983, ApJ, 273, L71

Fender, R. P., Hanson, M. M., Pooley, G. G. 1999, MNRAS, 308, 473

Eggleton, P. P. 1983, ApJ, 268, 368

Ghosh, P., Elsner, R. F., Weisskopf, M. C., \& Sutherland, P. G. 1981, ApJ, 251, 230

Giacconi, R., Gorenstein, P., Gursky, H. \& Waters, J. R. 1967, ApJ, 148, L119

Hanson, M. M., Still, M. D., \&Fender, R. P. 2000, ApJ, 541, 308

McCollough, M. L. et al. 2000, AAS/High Energy Astrophysics Division, 32, 28.05

Mioduszewski, A. J., Rupen, M. P., Hjellming, R. M., Pooley, G. G. \& Waltman, E. B. 2001, ApJ, 533, 766

Moffat, A. E. J., \& Marchenko, S. V. 1996, A\&A, 305, L29

Ogley, R. N., Bell Burnell, S. J., \& Fender, R. P. 2001, MNRAS, 322, 177

Paerels, F., Cottam, J., Sako, M., Liedahl, D. A., Brinkman, A. C., van der Meer, R. L. J., Kaastra, J. S. \& Predehl, P. 2000, ApJ, 533, L135

Predehl, P., Burwitz, V., Paerels, F., \& Trümper, J. 2000, A\&A, 357, L25 
Serlemitsos, P. J., Boldt, E. A., Holt, S. S., Rothschild, R. E., \& Saba, J. L. R. 1975, ApJ, 201, L9

Singh, N. S., Naik, S., Paul, B., Agrawal, P. C., Rao, A. R., \& Singh, K. Y. 2002, A\&A, 392, 161

Schmutz, W., Geballe, T. R. \& Schild, H. 1996, A\&A, 311, L25

Tavani, M., Ruderman, M., \& Shaham, J. 1989, ApJ, 342, L31

van der Klis, M. \& Bonnet-Bidaud, J. M. 1989, A\&A, 214, 203

van Kerkwijk, M. H. 1993, A\&A, 276, L9

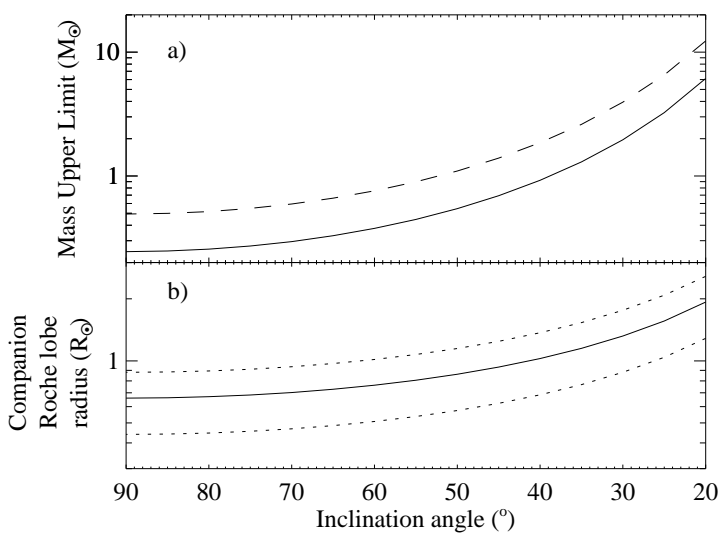

Fig. 3.- (a) Mass upper limits of the compact object (solid line) and the companion (dashed line) and (b) the Roche lobe radius of the companion (dotted lines are $1 \sigma$ limits) in the Cygnus X-3 system as a function of inclination angle. 